\title{
Conservative Treatment by Marsupialization of Dentigerous Cysts in Children: Report of three Cases
}

Durmuşlar $\mathrm{S}^{1 *}$, Durmuşlar $\mathrm{MC}^{2}$ and Nale $\mathbf{T}^{\mathbf{1}}$ ${ }^{1}$ Department of Pediatric Dentistry, Bülent Ecevit University, Turkey

${ }^{2}$ Department of Oral and Maxillofacial Surgery, Bülent Ecevit University, Turkey

*Corresponding author: Durmuşlar S, Department of Pediatric Dentistry, Faculty of Dentistry, Bülent Ecevit University, Esenköy, Zonguldak, Turkey

Received: J anuary 23, 2017; Accepted: February 25, 2017; Published: February 28, 2017

\begin{abstract}
Dentigerous cyst is the most common developmental odontogenic cyst. The standard treatment is enucleation of the cyst and extraction of the involved tooth. However, if the patient is a child, marsupialization may be advisable to allow eruption of a cyst-associated unerupted tooth, as a conservative and definitive treatment. This case report presents three cases of dentigerous cysts in children.
\end{abstract}

Two of the cases, lesions were detected at the right side of the mandible and associated with second primary molar and unerupted second premolar. One of the cases the lesion was detected at the right side of the maxilla and associated with first primary molar and unerupted first premolar. Patients were treated by extraction of the primary molars and marsupializing the cyst cavities. At 9 months follow up visits showed that the cystic lesions had resolved and the premolars were ready to erupt.

Because of the children have a great regenerative potential in bony structure and the teeth with incomplete root development maintain the eruptive strength, marsupialization is a conservative approach for treating dentigerous cysts in children.

Keywords: Dentigerous Cyst; Marsupialization; Unerupted tooth; Children

\section{Introduction}

Dentigerous cyst is benign and asymptomatic odontogenic cyst of the jaws. It is characterized by a unilocular radiolucent lesion that encloses or displaced unerupted teeth [1]. There are fluid accumulation between the reduced enamel epithelium and the crown with expansion of the tooth follicle [2-5]. This often causes cortical bone expansion, displacement of adjacent and involved teeth, resorption of teeth roots, tooth mobility and sensitivity if it reaches a size larger than $2 \mathrm{~cm}$ in diameter. Dentigerous cyst remain initially completely asymptomatic unless when infected [6,7].

Enucleation and marsupialization are the treatment options of dentigerous cysts. Marsupialization may be advisable for the involvement permanent tooth as cyts in children. This therapy allow the eruption of the permanent tooth involved. Because incomplete root development maintain the eruptive force $[2,3,8,9]$.

This case report presents three cases of dentigerous cysts which were associated with primary teeth caries in children. This article emphasizes that success of conservative approach of dentigerous cysts in children.

\section{Case Presentation}

\section{Case 1}

A 6-years-old girl was referred to the department of pediatric dentistry with an intermittent pain associated with tooth decay. Extraoral examination revealed no facial asymmetry. Intraoral examination revealed a hard submucosal mass and bone expansion of the buccal cortical plate of the alveolar ridge on the right side of the mandible which was caused deletion of the buccal folder. Radiographic examination showed a unilocular radiolucency surrounding the right mandibular primary and permanent molars and displacing the unerupted $2^{\text {nd }}$ premolar to the lower border of mandible (Figure 1A). At first, the contents of the swelling, serous and bloody liquid material, were aspirated and sent for histopathological evaluation. A provisional diagnosis of inflammatory type of dentigerous cyst was made on the basis of the clinical and radiographic findings.

Considering the age of the patient and the size of the cyst, marsupialization of the cystic cavity with preservation of all the involved permanent teeth was planned. After signing informed consent, the patient was treated by extraction of the right mandibular primary molars and marsupialization of the cystic cavity under local anesthesia. A plastic device made of anesthetic cartridges were inserted in the cystic cavity on the top of alveolar crest and sutured to the mucosa for creating a window with drain for irrigate the cystic cavity (Figure 1B).The drain was shortened as the cystic cavity volume was decreased.

\section{Case 2}

A 9-years-old boy was referred to the department of pediatric dentistry with an intermittent pain and swelling in the right mandible. Extraoral examination revealed facial asymmetry.Intraoral examination revealed a hard submucosal mass and bone expansion of the buccal cortical plate of the alveolar ridge on the right side of the mandible which was caused deletion of the buccal folder. Radiographic examination showed a unilocular radiolucency surrounding the unerupted 2nd premolar with a radiopaque border (Figure 2A). At first, the contents of the swelling, serous and bloody liquid
Austin J Dent - Volume 4 Issue 2 - 2017

ISSN : 2381-9189 | www.austinpublishing group.com

Durmuslar et al. (C) All rights are reserved 

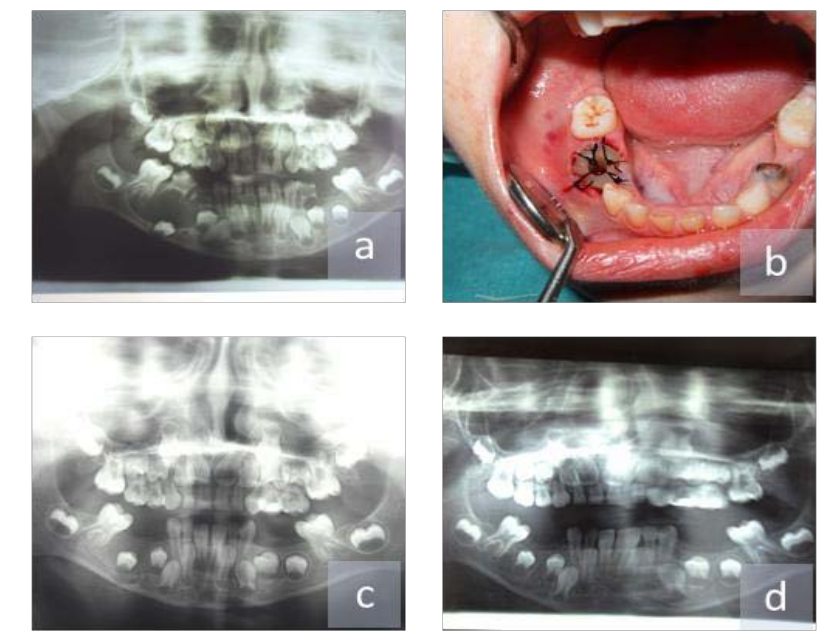

Figure 1: a) Initial panoramic radiograph b) Occlusal view after marsupialization of the cyst c) 2 months follow-up d) 9 months follow-up.
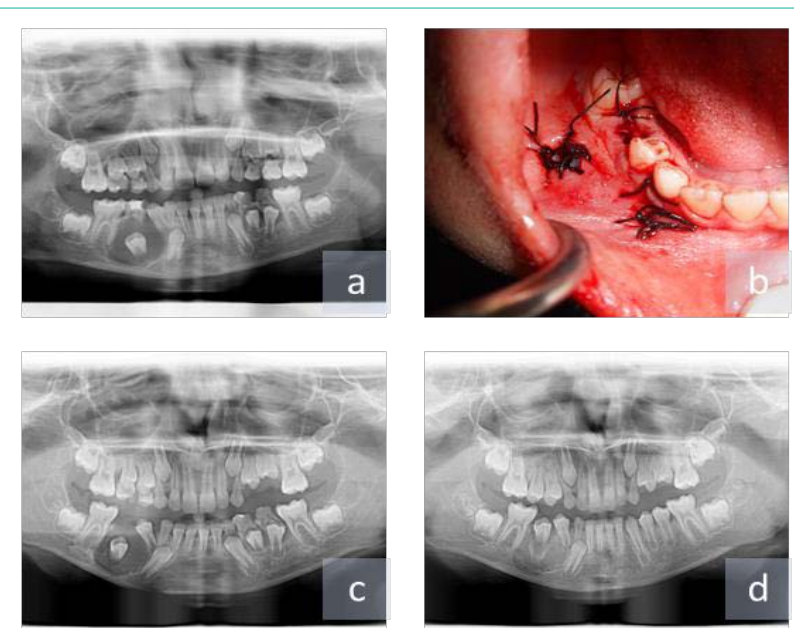

Figure 2: a) Initial panoramic radiograph b) Occlusal view after marsupialization of the cyst c) 2 months follow-up d) 9 months follow-up.

material, were aspirated and sent for histopathological evaluation. A provisional diagnosis of inflammatory type of dentigerous cyst was made on the basis of the clinical and radiographic findings.

Considering the age of the patient and the size of the cyst, marsupialization of the cystic cavity with preservation of all the involved permanent teeth was planned. After signing informed consent, the patient was treated by extraction of the right mandibular 2nd primary molar and marsupialization of the cystic cavity under local anesthesia. A buccal mucoperiosteal flap was elevated and the cyst membrane was fenestrated. The cyst membrane was sutured to oral mucosa to create a window of $1 \mathrm{x} 1 \mathrm{~cm}$ and a stent was inserted into the cystic lesion for irrigate the cystic cavity (Figure $2 \mathrm{~B}$ ). The stent couldn't be placed to the top of alveolar crest because of the tissue loss. The stent was shortened as the cystic cavity volume was decreased.

\section{Case 3}

A 7-years-old boy was referred to the department of pediatric dentistry for evaluation of pain that occurs when his mother kissing
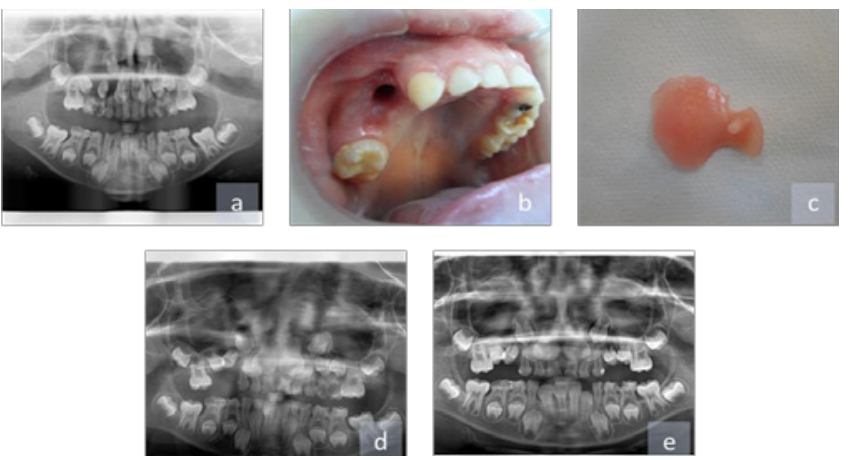

Figure 3: a) Initial panoramic radiograph b) Occlusal view after marsupialization of the cyst c) Obturator d) 2 months follow-up e) 9 months follow-up.

him on the right maxillar region. Extraoral examination revealed facial asymmetry. Intraoral examination revealed a hard submucosal mass and bone expansion of the buccal and palatal cortical plate of the alveolar ridge on the right side of the maxilla. Radiographic examination showed the radiolucency surrounding the unerupted maxillar right 1st premolar (Figure 3A). At first, the contents of the swelling, serous and bloody liquid material, were aspirated and sent for histopathological evaluation. A provisional diagnosis of inflammatory type of dentigerous cyst was made on the basis of the clinical and radiographic findings.

Considering the age of the patient and the size of the cyst, marsupialization of the cystic cavity with preservation of all the involved permanent teeth was planned. After signing informed consent, the patient was treated by extraction of the right maxillar primary molars and marsupialization of the cystic cavity under local anesthesia (Figure 3B). An obturator was designed to maintain wound patency after marsupialization. The obturator had a component that extended down into the cystic cavity through the top of alveolar crest and the component was shortened as the cystic cavity volume was decreased (Figure 3C).

Biopsy specimens were taken from the three of the patients and sent for histopathologic examination. Histologically, the epithelial lining with hyperplastic rete ridges were thick. According to the histologic diagnosis, the bony lesions were inflammatory types of dentigerous cysts.

After surgery, antibiotics (amoxicillin, 20-40 mg/kg per day in 3 doses) were prescribed for 5 days to prevent postoperative infection. Each of the parents were instructed to irrigate the cystic cavity through the wound holes with normal saline from a $10 \mathrm{ml}$ syringe with needle after each meal. On postoperative fifteenth day, temporary space maintainers were prepared for the Case 1 and Case 2. Patients were examined clinical and radiological month by month.

Radiographic decrease of lesion diameter and eruption of the involved premolars were achieved already in the first 2 months follow-up visits of the patients (Figure 1C,2C,3D). At the same time the shortened stents and obturator were completely removed because of the cavity contraction and contact to eruptive premolars and then actual space maintainers were prepared for the three patients. After 9 months, the cystic lesions had disappeared completely and the 
premolars were ready to erupt (Figure 1D,2D,3E). We have followed the patients and during this period, there have no recurrence of the cysts.

\section{Discussion}

There are 2 types of dentigerous cysts. The developmental type occurs in mature teeth, usually as a result of impaction. It is usually found in the late 2 nd and3rd decades and predominantly involve mandibular third molars. The inflammatory type occurs in immature teeth as a result of inflammation from a non vital primary tooth and subsequently spreading to the tooth follicle. It is found in the1st and early $2^{\text {nd }}$ decades [1]. Azaz and Shteyer [10] explained that persistent and prolonged inflammation may cause chronic irritation to the dental follicle of an unerupted tooth and this leads to development of a dentigerous cyst. The cases presented might be classified as inflammatory type, due to the lesions related with the carious primary molars and the patients' age. The results of histopathology also supports the diagnosis.

The standard treatment of the dentigerous cyst is enucleation of the cyst and extraction of the involved tooth. For large lesions this approach can cause fracture of the mandible or tooth devitalization. However, if patient is a child, marsupialization may be advisable to allow eruption of a cyst-associated unerupted tooth and inhibit possibility of pathologic fracture [5,9]. The choice of treatment depends on various factors, such as age, location and size of the cyst, tooth position in relation to the cyst, proximity to the vital structures and the degree of root formation [1]. Marsupialization consists of a surgical window on the wall of the cyst to relieve intracystic pressure. After marsupialization a stent, either a rubber tube or an obturator should be inserted into the cystic cavity opening to prevent spontaneous closure. This method has fewer complications than enucleation regarding the preservation of important anatomical structures and developing permanent tooth germs $[2,3,6,9]$.

In our cases, we choose conservative treatment based on the age of the patients and the strategic value of the associated teeth. The therapeutic option was curative, because of the children and their parents were very cooperative. In every bi-monthly follow up appointment, we observed the healing of the cyst and bone growth. The developing tooth germs emerged at proper position for eruption in just 9 months. It is important to affirm that no orthodontic appliance was necessary to assist the eruptive process. Hyomoto, et al. [11] were reported that $81 \%$ of dentigerous cyst associated mandibular premolars successfully erupted about 100 days after marsupialization without orthodontic traction. According to Yahara, et al. [12] that the successful eruption without orthodontic traction may be predicted 3 months after marsupialization. Also we observed radiographic decrease of the lesion diameter and the eruption of the involved premolars already in the first 2 months follow-up visits in all patients.

Previous studies have also indicated that the age of patient affects the successful eruption of a dentigerous cyst associated premolar. Accordingly, when the age of patients are between 7-12 years the eruption potential is good, but between 11-16 years are not [11,12]. Therefore the ages of our cases has been appropriate for the eruption potential.

If untreated, these cysts may cause pathologic bone fracture, impaction of the permanent tooth, bone deformation, ameloblastoma and development of oral squamous cell carcinoma or mucoepidermoid carcinoma [3,7]. Due to the children have smaller jaws than adults, especially the bone fracture risk increases in children post-treatment, if the cyst was enucleated [13]. So, because of the children have a great regenerative potential in bony structure and the teeth with incomplete root development maintain the eruptive strength, marsupialization is a conservative approach for treating dentigerous cysts in children $[2,4,9]$.

\section{Conclusion}

The diagnosis and early treatment of dentigerous cysts in children is very important, especially in cases where the lesions enclose permanent teeth in order to minimize the associated damages, such as those presented in this article. According to cystic size, age of the patient, closeness to vital structures and the strategic value of the impacted tooth, conservative treatment is a favorable treatment modality for extensive dentigerous cysts. The successful preservation and eruption of the affected teeth in this report supports conservative management of dentigerous cysts in children.

\section{References}

1. Bhardwaj B, Sharma S, Chitlangia P, Agarwal P, Bhamboo A, Rastogi K. Mandibular Dentigerous Cyst in a 10 -Year-Old Child. Int J Clin Pediatr Dent. 2016; 9: 281-284.

2. $\mathrm{Hu} \mathrm{YH}$, Chang $\mathrm{YL}$, Tsai $\mathrm{A}$. Conservative treatment of dentigerous cyst associated with primary teeth. Oral Surg Oral Med Oral Pathol Oral Radiol Endod. 2011; 112: e5-7.

3. Kirtaniya BC, Sachdev V, Singla A, Sharma AK. Marsupialization: a conservative approach for treating dentigerous cyst in children in the mixed dentition. J Indian Soc Pedod Prev Dent. 2010; 28: 203-208.

4. Martinez-Perez D, Varela-Morales M. Conservative treatment of dentigerous cysts in children: a report of 4 cases. J Oral Maxillofac Surg. 2001; 59: 331333.

5. Arjona-Amo M, Serrera-Figallo MA, Hernández-Guisado JM, Gutiérrez-Pérez $\mathrm{JL}$, Torres-Lagares D. Conservative management of dentigerous cysts in children. J Clin Exp Dent. 2015; 7: e671-674.

6. Ertas U, Yavuz MS. Interesting eruption of 4 teeth associated with a large dentigerous cyst in mandible by only marsupialization. J Oral Maxillofac Surg. 2003; 61: 728-730.

7. Hegde RJ, Khare SS, Devrukhkar VN. Dentigerous cyst in a young child: clinical insight and a case report. J Indian Soc Pedod Prev Dent. 2013; 31: 209-211.

8. Benn A, Altini M. Dentigerous cysts of inflammatory origin. A clinicopathologic study. Oral Surg Oral Med Oral Pathol Oral Radiol Endod. 1996; 81: 203-209.

9. Carrera M, Dantas DB, Marchionni AM, de Oliveira MG, Andrade MG. Conservative treatment of the dentigerous cyst: report of two cases. Braz J Oral Sci. 2013; 12: 52-56.

10. Azaz B, Shteyer A. Dentigerous cysts associated with second mandibular bicuspids in children: report of five cases. ASDC J Dent Child. 1973; 40: 2931.

11. Hyomoto M, Kawakami M, Inoue M, Kirita T. Clinical conditions for eruption of maxillary canines and mandibular premolars associated with dentigerous cysts. Am J Orthod Dentofacial Orthop. 2003; 124: 515-520.

12. Yahara $Y$, Kubota $Y$, Yamashiro $T$, Shirasuna K. Eruption prediction of mandibular premolars associated with dentigerous cysts. Oral Surg Oral Med Oral Pathol Oral Radiol Endod. 2009; 108: 28-31.

13. Berti Sde A, Pompermayer AB, Couto Souza PH, Tanaka OM, Westphalen VP, Westphalen FH. Spontaneous eruption of a canine after marsupialization of an infected dentigerous cyst. Am J Orthod Dentofacial Orthop. 2010; 137 : 690-693. 